\title{
Is there an optimal "door to cath time" in the treatment of acute pulmonary embolism with catheter-directed thrombolysis?
}

\author{
Aranyak Rawal ${ }^{1}$, Devarshi Ardeshna ${ }^{2}$, Kirstin Hesterberg ${ }^{3}$, Brandon Cave $^{4}$, Uzoma N. Ibebuogu ${ }^{3}$, \\ Rami N. Khouzam ${ }^{3}$
}

${ }^{1}$ Department of Internal Medicine-Pediatrics, ${ }^{2}$ College of Medicine, University of Tennessee Health Science Center, Memphis, TN, USA; ${ }^{3}$ Division of Cardiovascular Diseases, Department of Internal Medicine, University of Tennessee Health Science Center, Memphis, TN, USA; ${ }^{4}$ Department of Pharmacy, Methodist University Hospital, Memphis, TN, USA

Contributions: (I) Conception and design: A Rawal, D Ardeshna, K Hesterberg, B Cave; (II) Administrative support: RN Khouzam; (III) Provision of study materials or patients: All authors; (IV) Collection and assembly of data: All authors; (V) Data analysis and interpretation: All authors; (VI) Manuscript writing: All authors; (VII) Final approval of manuscript: All authors.

Correspondence to: Aranyak Rawal, MD. Department of Internal Medicine-Pediatrics, University of Tennessee Health Science Center, Memphis, TN 38163, USA. Email: arawal1@uthsc.edu.

\begin{abstract}
Ultrasound assisted catheter-directed thrombolysis (UACT) is a relatively novel approach to treating acute pulmonary embolism (PE). It is an alternative to systemic thrombolysis with good success rates and low reported in-hospital mortality, and low rates of procedure-related major and minor bleeding. Since UACT received FDA approval for the treatment of PE in 2014, there is paucity of data regarding the optimal timing of initiation of the procedure after the initial diagnosis is made. We reviewed the available literature regarding UACT for acute PE and found six studies that included time to procedure. Based on our review, patients may benefit from early (<24-48 h after presentation) rather than delayed ( $>48 \mathrm{~h})$ initiation. Early initiation of therapy has shown to improve pulmonary arterial pressures, right ventricular (RV) to left ventricular (LV) ratios, with low rates of bleeding and low post procedural and in hospital mortality. However, further studies are required to confirm these findings and establish the appropriate timeline for initiation of UACT.
\end{abstract}

Keywords: Acute pulmonary embolism (PE); ultrasound assisted catheter-directed thrombolysis (UACT);
catheter-directed thrombolysis (CDT); Ekosonic endovascular system (EKOS), Time to ultrasound assisted
catheter-directed thrombolysis (UACT)

Submitted Jul 30, 2019. Accepted for publication Jul 23, 2019.

doi: 10.21037/atm.2019.07.89

View this article at: http://dx.doi.org/10.21037/atm.2019.07.89

\section{Introduction}

Pulmonary embolism (PE) is the third leading cause of cardiovascular death, after heart attack and stroke (1). The management of $\mathrm{PE}$ varies depending on the stratification of low, intermediate or high-risk (2). High-risk or "massive" PE is characterized by hemodynamic instability. Intermediate risk or "submassive" PE is hemodynamically stable, however, there is evidence of right ventricular (RV) dysfunction determined by imaging or elevated cardiac biomarkers. Finally, low-risk PE is neither hemodynamically unstable nor is there evidence of RV dysfunction (3). Patients presenting with high-risk PE have in-hospital mortality rates up to $52 \%$, whereas intermediate-risk and low-risk PE mortality rates of up to $15 \%$ and less than $5 \%$, respectively (4).

Systemic thrombolysis remains the recommended therapy in the treatment of high-risk PE and can provide rapid improvement in $\mathrm{RV}$ dilation, a reduction in clot burden, and return to hemodynamic stability (4). Despite 
these benefits, systemic thrombolysis is reported to be underutilized and withheld in up to $68 \%$ percent of eligible patients. (5). Avoidance of fibrinolytics are frequently due to bleeding concerns, uneasiness with use, contraindications to systemic thrombolysis, and the advent of percutaneous techniques. Additionally, the use of systemic thrombolysis in the management of intermediate-risk PEs remains controversial and is not currently recommended (6-9). A relatively new intervention, catheter-directed thrombolysis (CDT) has been successfully performed acutely in both high and intermediate-risk patients with low in-hospital mortality, and low rates of procedure-related major and minor bleeding.

Following its FDA approval in 2014, ultrasound assistance in combination with CDT is often utilized in the management of acute PE. Ultrasound assisted catheterdirected thrombolysis (UACT) utilizes high frequency, lowpower ultrasound waves to improve thrombus penetration by thrombolytic medications, thus causing temporary fibrin molecule separation and exposure of binding sites for the thrombolytic medications $(10,11)$. In this fashion, UACT allows for direct administration of thrombolytic medications to the clot location. From a clinical standpoint, this translates to a lower risk of bleeding compared to systemic thrombolytic therapy while providing a similar therapeutic benefit (12). While there have been a number of studies that evaluate the clinical outcomes of UACT $(7,13-30)$, there is a paucity of data regarding the optimal timing of therapy initiation. Current consensus guidelines do not state when UACT should be initiated after the initial diagnosis of acute high or intermediate-risk PE (3). The goal of this review is to describe the current literature available in regard to timing of UACT after diagnosis of PE.

\section{Methods}

A search of the PubMed database was performed to identify studies of patients with acute, high or intermediate-risk PE from January 1990 to May 2019. Our search method used combined text words and medical subject headings (MeSH). The keywords used included: "acute pulmonary embolism catheter thrombolysis", "catheter directed thrombolysis", "acute pulmonary embolism and catheter directed thrombolysis timing", "acute pulmonary embolism and ultrasound assisted thrombolysis." We also cross-referenced sources in target articles for additional references. All studies included were published in English.

\section{Study selection and data extraction}

Following review of the PubMed database results, we included articles if (I) they were either a randomized controlled trial, retrospective, or prospective study of patients who underwent UACT for acute PE; (II) included time from initial diagnosis of PE to CDT; (III) included only patients who were classified as having acute highrisk and/or intermediate-risk PE; (IV) included total number of patients who underwent CDT; (V) included bleeding outcomes. Final inclusion of articles was based on consensus by authors. We report the following data from the studies: number of patients included in the study, reported time from procedure to intervention, number of patients that were classified as high or intermediaterisk PE, bleeding rate, dose of thrombolytic utilized, and average hospital stay.

\section{Results}

Our MeSH search strategy resulted in a total of 329 articles. Of the 329 studies, 308 were excluded based on article type. As a result, 21 prospective, retrospective and randomized clinical trials were assessed for eligibility. Of these, 16 were excluded because they did not include information on timing of the procedure relative to diagnosis of $\mathrm{PE}(6,13-18$, 20,21,24-30). Six studies were analyzed for our final review and have been summarized in Table 1. Four studies, (Edla et al., Gaba et al., Voore et al., Lee et al.) were retrospective studies and two studies [Tapson et al. (31), Kucher et al.] were randomized controlled trials.

Edla $e t a l$. is one of two studies to date that directly compared the effect of timing of UACT in the setting of acute PE (32). The study included 41 patients with acute intermediate-risk $\mathrm{PE}$, of which 21 patients received early ( $<24 \mathrm{~h}$ from diagnosis) UACT, and 20 received delayed $(>24 \mathrm{~h})$ UACT. The average time to procedure for the early group and delayed group was $13.3 \pm 5.6 \mathrm{~h}$ and $46.4 \pm 10.1 \mathrm{~h}$, respectively. $\mathrm{RV}$ to left ventricular $(\mathrm{LV})$ ratio, mean pulmonary arterial pressure, cardiac index, pulmonary vascular resistance, RV stroke index, pulmonary artery pulsatility index, in-hospital all-cause mortality, recurrent PE, bleeding, procedurerelated complications, and post-procedure length of stay were compared. The study found a significant improvement in cardiac index $\left(0.6\right.$ vs. $\left.0.4 \mathrm{~L} / \mathrm{min} / \mathrm{m}^{2} ; \mathrm{P}<0.001\right)$, pulmonary vascular resistance $(3.4$ vs. 0.5 Wood units; $\mathrm{P}<0.001)$ and mean RV stroke work index $\left(3.5 \pm 2.0\right.$ vs. $2.3 \pm 1.5 \mathrm{gm} / \mathrm{mg}^{2}$ per beat; $\mathrm{P}=0.04)$ in the early compared to the delayed group. 


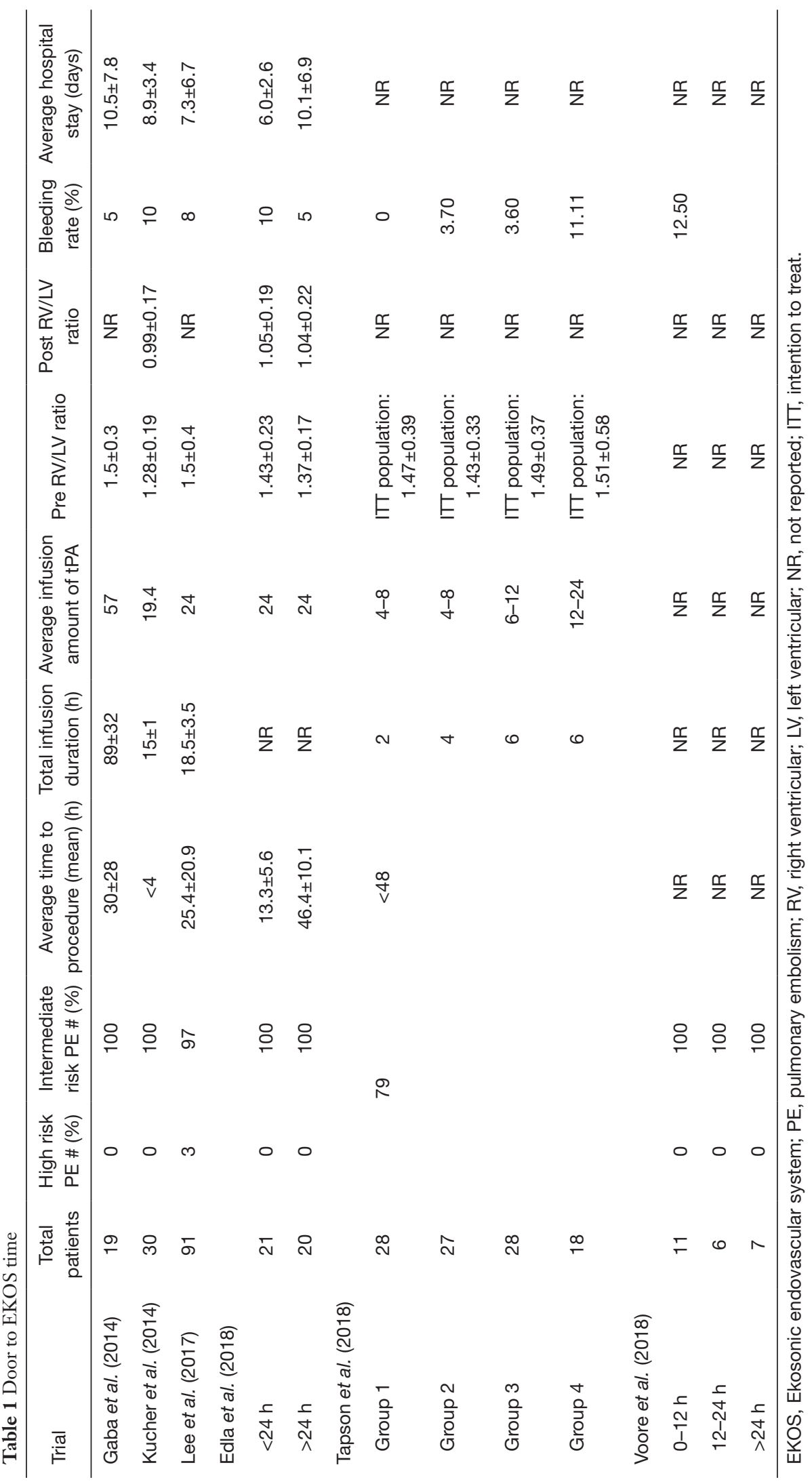


Additionally, there was a trend in favor of early treatment in the mean improvement in $\mathrm{RV} / \mathrm{LV}$ ratio $(0.38 \pm 0.17 v s$. $0.33 \pm 0.21 ; \mathrm{P}=0.40)$. No GUSTO-defined major bleeding or in-hospital mortality was reported.

Voore et al. also conducted an analysis to evaluate the timing of UACT on outcomes (33). In a small retrospective study of 24 patients with acute intermediate-risk PE, patients were classified into three groups consisting of those who underwent UACT after diagnosis of PE between $0-12 \mathrm{~h}$, 12-24 h, and $>24 \mathrm{~h}$ after diagnosis. The study found no statistical difference between the three groups for the outcomes reported. Specifically, there was no difference between the time of initiation of UACT and the resolution of oxygen requirements $(\mathrm{P}=0.52)$, decrease in heart rate $(\mathrm{P}=0.27)$, mortality $(\mathrm{P}=1.00)$, and bleeding $(\mathrm{P}=0.54)$. The study reported no echocardiographic, invasive hemodynamic parameters. A total of three deaths occurred in the study, one from each group.

The Ultrasound Accelerated Thrombolysis of Pulmonary Embolism (ULTIMA) trial compared intermediate-risk patients randomized to either UACT or heparin-based therapy (7). Eligible patients had a diagnosis of acute, symptomatic PE of at least one main or proximal lower lobe artery and an RV/LV ratio of greater than or equal to one. The primary outcome was the change in $\mathrm{RV} / \mathrm{LV}$ ratio at $24 \mathrm{~h}$. Additional outcomes included major and minor bleeding, recurrent venous thromboembolism, death, hemodynamic decompensation, echocardiographic parameters, and invasive hemodynamic parameters. A total of 59 patients with acute intermediate-risk PE were included; 30 patients in the UACT group and 29 patients in the heparin group. All patients had UACT initiated within $4 \mathrm{~h}$ of echocardiographic identification of right ventricular dilation. The trial showed a significant improvement in RV/ $\mathrm{LV}$ ratio at $24 \mathrm{~h}$ with UACT compared to heparin therapy $(\Delta 0.30 \pm 0.20$ vs. $\Delta 0.03 \pm 0.16 ; \mathrm{P}<0.001)$. Additionally, there was significant improvement in hemodynamic parameters in the UACT group when compared with the heparin group. Invasive hemodynamic monitoring showed statistically significant improvement in pulmonary artery systolic, diastolic and mean pressure, right atrial mean pressure, and cardiac index at $18 \pm 3 \mathrm{~h}$ after procedure. There were no episodes of major bleeding in either group. At 90-day follow up, there was not a significant difference in RV/LV ratio, although there was a trend favoring UACT. At 90-day follow up, there were no difference in rates of hemodynamic decompensation, recurrent VTE or major bleeding between the UACT and standard therapy groups.
A randomized trial of the Optimum Duration of Acoustic Pulse Thrombolysis Procedure in Acute Intermediate-Risk Pulmonary Embolism (OPTALYSE PE) trial evaluated lowest optimal dose and duration for intermediate, acute $\mathrm{PE}$ patients. This randomized, prospective trial enrolled 101 patients with mean age of 60 years who presented with $\mathrm{PE}$ symptoms for $<14$ days, $>90 \mathrm{mmHg}$ systolic blood pressure, RV/LV diameter $\geq 0.9$ and PE located in at least 1 main or proximal lobar pulmonary artery. All patients received UACT within $48 \mathrm{~h}$ of diagnosis. The patients were randomized to 4 groups of UACT with various regimens of tPA dose per catheter: $2 \mathrm{mg} / \mathrm{h}$ for $2 \mathrm{~h}, 1 \mathrm{mg} / \mathrm{h}$ for $4 \mathrm{~h}, 1 \mathrm{mg} / \mathrm{h}$ for $6 \mathrm{~h}, 2 \mathrm{mg} / \mathrm{h}$ for $6 \mathrm{~h}$. RV/LV ratio measured at $48 \pm 6 \mathrm{~h}$ after start of UACT reduced by $24.0 \% \pm 15.9 \%(\mathrm{P}<0.0001)$, $22.6 \% \pm 14.1 \%(\mathrm{P}<0.0001), 26.3 \% \pm 16.8 \%(\mathrm{P}<0.0001)$ and $25.5 \% \pm 22.7 \%(\mathrm{P}=0.0011)$ compared to baseline in the intention-to-treat population. Major bleeding, as defined by International Society on Thrombosis and Hemostasis, was calculated at $72 \mathrm{~h}$ after the start of UACT and was reported in $4 \%$ of patients. No bleeding events were reported in Group 1. Group 2 and Group 3 each had a patient with bleeding, which was related to either systemic or higher tPA dose. Randomization to Group 4 was stopped after a patient developed fatal ICH, which was attributed to UACT and the only death in the study. Recurrent PE was noted in one patient in Group 3, attributed to non-compliance with his anticoagulation. A significant limitation of the study is the limited sample between regimens and a lack of a control group. Despite this, it appears that lower doses and shorter durations are effective in improving RV/LV and have low risk of bleeding.

Lee et al. (22) was a retrospective study that included 91 patients who underwent UACT over the course of 39 months. Patients were included if they had a diagnosis of symptomatic, acute $\mathrm{PE}$, with $\mathrm{RV} / \mathrm{LV}$ ratio greater than one. Reported outcomes included changes in pulmonary arterial pressures, bleeding and average length of hospital stay. The average time from presentation to catheter intervention was $25.4 \pm 20.9$ h. Of the 91 patients enrolled, 88 patients were classified as having intermediate-risk $\mathrm{PE}$ while three were classified as high-risk PE. The study found a significant reduction in pulmonary arterial pressures from an average of $56.1 \pm 15.2 \mathrm{mmHg}$ pre-therapy to $34.3 \pm 15.2 \mathrm{mmHg}$ after UACT. Seven patients (8\%) experienced major bleeding while 14 patients (15\%) experienced minor bleeding. Interestingly, of the 17 patients included that were high-risk for thrombolysis and systemic anticoagulation there were three major bleeding events, which was not significantly 
higher than the lower risk group.

Gaba et al. retrospectively reviewed 19 patients with acute intermediate-risk PE who underwent CDT between 2009 and 2013 (19). The average presentation to catheter intervention time was $30 \pm 28 \mathrm{~h}$ with a range of four to $96 \mathrm{~h}$. The study reported outcomes of procedural and clinical success (defined as resolution of symptoms), 30 and 90-day survival, and survival to hospital discharge. The authors reported $100 \%$ procedural and clinical success, a $95 \%$ 30 -day and 94\% 90-day survival, and 95\% survival to discharge. One patient experienced a fatal bleed. This study was limited by its small sample size. No echocardiographic or hemodynamic parameters were reported, limiting comparison to the previously described studies. Additionally, ultra-sound assistance was not used in this study.

\section{Discussion}

An increase of the RV/LV ratio is an important predictor of in-hospital mortality for patients presenting with acute PE (34). The ULTIMA and SEATTLE-II trials both demonstrated that UACT is an effective alternative to standard therapy for reducing the RV/LV ratio in patients with acute intermediate-risk PEs, without significantly increased major bleeding complications (28). Current guidelines recommend use of CDT with or without ultrasound assistance on a case to case basis due to the lack of randomized clinical data available (3). However, there are several trials underway that aim to expand the available knowledge regarding UACT for the treatment of acute PE (35-37). As more information becomes available, this may lead to increased utilization of UACT for the treatment of high and intermediate-risk PE. However, there continues to be a lack of studies evaluating the optimal time from diagnosis to initiation of UACT. As such, there remains a lack of consensus as to the optimal time to procedure.

The optimal time from diagnosis to initiation of thrombolytic or anticoagulation therapy is not well established either. Early trials of thrombolysis found no significant difference in reduction of clot burden for patients who received thrombolysis within two days of symptom onset compared to patients who received thrombolysis between 3-5 days from symptom onset (38-40). However, a more recent study showed that for each additional day of symptoms prior to thrombolysis, there was a $0.8 \%$ decrease in lung tissue perfusion on reperfusion imaging (41). In addition, evidence exists that patients presenting with cardiac arrest or with high-risk PE had an improved in- hospital mortality and less recurrent $\mathrm{PE}$ if thrombolytic therapy was initiated within $1 \mathrm{~h}$ of diagnosis (42).

In our review, Edla et al. was the largest study to directly compare the outcomes of early versus delayed CDT initiation (32). While there was improvement in pulmonary arterial pressures compared to delayed CDT therapy, RV/LV ratio change was not significantly different. Voore et al. was a similar retrospective study to directly compare outcomes based on the timing of UACT (33). They stratified patients into three groups based on procedure timing $(0-12,12-24$, and $>24 \mathrm{~h})$ and found no statistically significant difference in outcomes. However, results were limited small sample size and no reported echocardiographic parameter outcomes. The ULTIMA study initiated CDT therapy within $4 \mathrm{~h}$ of baseline echocardiography and showed improvement in RV/LV ratio compared to standard therapy (7). It showed that UACT is an effective alternative to systemic heparin therapy alone, with improved short term echocardiographic and hemodynamic parameters. It is unclear if this benefit persists at longer term follow up. OPTALYSE PE showed improvement in $\mathrm{RV} / \mathrm{LV}$ ratio with shorter duration and lower dose of tPA with CDT done within $48 \mathrm{~h}$ of diagnosis. The other two retrospective studies, both had an average of $>24 \mathrm{~h}$ from time of presentation to initiation of CDT therapy but did not report the RV/LV ratio $(19,22)$.

Despite the variations in reported outcomes in these studies, there is a trend towards benefit in early interventions. Edla et al., ULTIMA, and Lee et al. all reported improvement in mean pulmonary arterial pressure with early intervention $(7,22,32)$. The ULTIMA study showed that intervention within $4 \mathrm{~h}$ of $\mathrm{PE}$ diagnosis improved RV/LV significantly compared to heparin alone (7). While early intervention was associated with increased resolution of PE, Voore $e t$ al. were not able to replicate this finding and regrettably, limited hemodynamic parameters prevent effective comparison. Lee et al. and Gaba et al. did not stratify the patient outcomes based on timing. However, both studies initiated CDT within an average of $30 \mathrm{~h}$ and reported good PE resolution with low rates of bleeding.

There are significant limitations in the ability to generate conclusions from the available literature. First, the variations in CDT treatment regimens and reported outcomes does not allow for adequate comparisons. While some studies report $\mathrm{PE}$ resolution as $\mathrm{RV} / \mathrm{LV}$ ratio, others report pulmonary artery pressure. Furthermore, the average time of CDT initiation ranges from less than $5 \mathrm{~h}$ to more than $50 \mathrm{~h}$ from $\mathrm{PE}$ diagnosis. The infusion 
duration ranges from $2 \mathrm{~h}$ to over $100 \mathrm{~h}$ and the tPA dosing ranges from 4 to $57 \mathrm{mg}$ are confounding factors in efforts to determine optimal timing. Amidst such variation, there is a potential for benefit with early intervention yet will require confirmation in larger studies. Second, a majority of the studies do not describe the timeline of CDT initiation or a mean time to procedure. Of our review of six studies, many consisted of small patient populations, in which positive trends in outcomes are encouraging for early intervention. While the majority of patients studied have been classified as intermediate risk, perhaps a door-tocath time requirement may be more impactful in high-risk patients who are hemodynamically unstable and likely to benefit from earlier intervention.

\section{Conclusions}

While catheter directed thrombolysis is an effective management strategy for acute $\mathrm{PE}$, there is no consensus available for the optimal timing of initiation of therapy. Based on review of the available literature, UACT may provide benefit for patients started on therapy early $(<24-48$ after presentation) compared to those in whom UACT is delayed. However, further studies are needed to further clarify the role and benefit of UACT.

\section{Acknowledgments}

None.

\section{Footnote}

Conflicts of Interest: The authors have no conflicts of interest to declare.

Ethical Statement: The authors are accountable for all aspects of the work in ensuring that questions related to the accuracy or integrity of any part of the work are appropriately investigated and resolved.

\section{References}

1. Heit JA. The epidemiology of venous thromboembolism in the community: implications for prevention and management. J Thromb Thrombolysis 2006;21:23-9.

2. Konstantinides SV. 2014 ESC Guidelines on the diagnosis and management of acute pulmonary embolism. Eur Heart J 2014;35:3145-6.
3. Jaff MR, McMurtry MS, Archer SL, et al. Management of massive and submassive pulmonary embolism, iliofemoral deep vein thrombosis, and chronic thromboembolic pulmonary hypertension: a scientific statement from the American Heart Association. Circulation 2011;123:1788-830.

4. Torbicki A, Perrier A, Konstantinides S, et al. Guidelines on the diagnosis and management of acute pulmonary embolism: the Task Force for the Diagnosis and Management of Acute Pulmonary Embolism of the European Society of Cardiology (ESC). Eur Heart J 2008;29:2276-315.

5. Zuin M, Rigatelli G, Zuliani G, et al. Thrombolysis in hemodynamically unstable patients: still underused: a review based on multicenter prospective registries on acute pulmonary embolism. J Thromb Thrombolysis 2019;48:323-30.

6. Avgerinos ED, Abou Ali AN, Liang NL, et al. Catheterdirected interventions compared with systemic thrombolysis achieve improved ventricular function recovery at a potentially lower complication rate for acute pulmonary embolism. J Vasc Surg Venous Lymphat Disord 2018;6:425-32.

7. Kucher N, Boekstegers P, Müller OJ, et al. Randomized, controlled trial of ultrasound-assisted catheter-directed thrombolysis for acute intermediate-risk pulmonary embolism. Circulation 2014;129:479-86.

8. Patel N, Patel NJ, Agnihotri K, et al. Utilization of catheter-directed thrombolysis in pulmonary embolism and outcome difference between systemic thrombolysis and catheter-directed thrombolysis. Catheter Cardiovasc Interv 2015;86:1219-27.

9. Stein PD, Matta F. Thrombolytic therapy in unstable patients with acute pulmonary embolism: saves lives but underused. Am J Med 2012;125:465-70.

10. Braaten JV, Goss RA, Francis CW. Ultrasound reversibly disaggregates fibrin fibers. Thromb Haemost 1997;78:1063-8.

11. Siddiqi F, Odrljin TM, Fay PJ, et al. Binding of tissueplasminogen activator to fibrin: effect of ultrasound. Blood 1998;91:2019-25.

12. Kaymaz C, Akbal OY, Tanboga IH, et al. UltrasoundAssisted Catheter-Directed Thrombolysis in High-Risk and Intermediate-High-Risk Pulmonary Embolism: A Meta-Analysis. Curr Vasc Pharmacol 2018;16:179-89.

13. Bagla S, Smirniotopoulos JB, van Breda A, et al. Ultrasound-accelerated catheter-directed thrombolysis for acute submassive pulmonary embolism. J Vasc Interv 
Radiol 2015;26:1001-6.

14. Bloomer TL, El-Hayek GE, McDaniel MC, et al. Safety of catheter-directed thrombolysis for massive and submassive pulmonary embolism: Results of a multicenter registry and meta-analysis. Catheter Cardiovasc Interv 2017;89:754-60.

15. Dumantepe M, Uyar I, Teymen B, et al. Improvements in pulmonary artery pressure and right ventricular function after ultrasound-accelerated catheter-directed thrombolysis for the treatment of pulmonary embolism. J Card Surg 2014;29:455-63.

16. Engelberger RP, Moschovitis A, Fahrni J, et al. Fixed lowdose ultrasound-assisted catheter-directed thrombolysis for intermediate and high-risk pulmonary embolism. Eur Heart J 2015;36:597-604.

17. Engelhardt TC, Taylor AJ, Simprini LA, et al. Catheterdirected ultrasound-accelerated thrombolysis for the treatment of acute pulmonary embolism. Thromb Res 2011;128:149-54.

18. Fuller TJ, Paprzycki CM, Zubair MH, et al. Initial Experiences with Endovascular Management of Submassive Pulmonary Embolism: Is It Safe? Ann Vasc Surg 2017;38:158-63.

19. Gaba RC, Gundavaram MS, Parvinian A, et al. Efficacy and safety of flow-directed pulmonary artery catheter thrombolysis for treatment of submassive pulmonary embolism. AJR Am J Roentgenol 2014;202:1355-60.

20. Kennedy RJ, Kenney HH, Dunfee BL. Thrombus resolution and hemodynamic recovery using ultrasoundaccelerated thrombolysis in acute pulmonary embolism. J Vasc Interv Radiol 2013;24:841-8.

21. Kuo WT, Banerjee A, Kim PS, et al. Pulmonary Embolism Response to Fragmentation, Embolectomy, and Catheter Thrombolysis (PERFECT): Initial Results From a Prospective Multicenter Registry. Chest 2015;148:667-73.

22. Lee KA, Cha A, Kumar MH, et al. Catheter-directed, ultrasound-assisted thrombolysis is a safe and effective treatment for pulmonary embolism, even in highrisk patients. J Vasc Surg Venous Lymphat Disord 2017;5:165-70.

23. Lin PH, Annambhotla S, Bechara CF, et al. Comparison of percutaneous ultrasound-accelerated thrombolysis versus catheter-directed thrombolysis in patients with acute massive pulmonary embolism. Vascular 2009;17 Suppl 3:S137-47.

24. McCabe JM, Huang PH, Riedl L, et al. Usefulness and safety of ultrasound-assisted catheter-directed thrombolysis for submassive pulmonary emboli. Am J Cardiol 2015;115:821-4.
25. Nykamp M, VandenHull A, Remund T, et al. Safety and efficacy of ultrasound-accelerated catheter-directed lytic therapy in acute pulmonary embolism with and without hemodynamic instability. J Vasc Surg Venous Lymphat Disord 2015;3:251-7.

26. Ozcinar E, Cakici M, Dikmen Yaman N, et al. Thrombus resolution and right ventricular functional recovery using ultrasound-accelerated thrombolysis in acute massive and submassive pulmonary embolism. Int Angiol 2017;36:428-37.

27. Ozmen C, Deniz A, Akilli RE, et al. Ultrasound Accelerated Thrombolysis May Be an Effective and Safe Treatment Modality for Intermediate Risk/Submassive Pulmonary Embolism. Int Heart J 2016;57:91-5.

28. Piazza G, Hohlfelder B, Jaff MR, et al. A Prospective, Single-Arm, Multicenter Trial of Ultrasound-Facilitated, Catheter-Directed, Low-Dose Fibrinolysis for Acute Massive and Submassive Pulmonary Embolism: The SEATTLE II Study. JACC Cardiovasc Interv 2015;8:1382-92.

29. Quintana D, Salsamendi J, Fourzali R, et al. Ultrasoundassisted thrombolysis in submassive and massive pulmonary embolism: assessment of lung obstruction before and after catheter-directed therapy. Cardiovasc Intervent Radiol 2014;37:420-6.

30. Yoo JW, Choi HC, Lee SJ, et al. Comparison between systemic and catheter thrombolysis in patients with pulmonary embolism. Am J Emerg Med 2016;34:985-8.

31. Tapson VF, Sterling K, Jones N, et al. A Randomized Trial of the Optimum Duration of Acoustic Pulse Thrombolysis Procedure in Acute Intermediate-Risk Pulmonary Embolism: The OPTALYSE PE Trial. JACC Cardiovasc Interv 2018;11:1401-10.

32. Edla S, Rosman H, Neupane S, et al. Early Versus Delayed Use of Ultrasound-Assisted Catheter-Directed Thrombolysis in Patients With Acute Submassive Pulmonary Embolism. J Invasive Cardiol 2018;30:157-62.

33. Voore P, Suárez JMV, Dereddi R, et al. Optimal Timing of Catheter-Directed Thrombolysis in Treatment of Submassive Pulmonary Embolism. Crit Care Med 2018;46:66.

34. Frémont B, Pacouret G, Jacobi D, et al. Prognostic value of echocardiographic right/left ventricular end-diastolic diameter ratio in patients with acute pulmonary embolism: results from a monocenter registry of 1,416 patients. Chest 2008;133:358-62.

35. Storey RF, Angiolillo DJ, Patil SB, et al. Inhibitory Effects of Ticagrelor Compared With Clopidogrel on Platelet 
Function in Patients With Acute Coronary Syndromes: The PLATO (PLATelet inhibition and patient Outcomes) PLATELET Substudy. J Am Coll Cardiol 2010;56:1456-62.

36. Montalescot G, Bolognese L, Dudek D, et al. Pretreatment with Prasugrel in Non-ST-Segment Elevation Acute Coronary Syndromes. N Engl J Med 2013;369:999-1010.

37. Parodi G, Valenti R, Bellandi B, et al. Comparison of Prasugrel and Ticagrelor Loading Doses in ST-Segment Elevation Myocardial Infarction Patients: RAPID (Rapid Activity of Platelet Inhibitor Drugs) Primary PCI Study. J Am Coll Cardiol 2013;61:1601-6.

38. Goldhaber SZ, Markis JE, Kessler CM, et al. Perspectives on treatment of acute pulmonary embolism with tissue plasminogen activator. Semin Thromb Hemost 1987;13:171-7.

Cite this article as: Rawal A, Ardeshna D, Hesterberg K, Cave B, Ibebuogu UN, Khouzam RN. Is there an optimal "door to cath time" in the treatment of acute pulmonary embolism with catheter-directed thrombolysis? Ann Transl Med 2019;7(17):419. doi: 10.21037/atm.2019.07.89
39. Goldhaber SZ, Meyerovitz MF, Markis JE, et al. Thrombolytic therapy of acute pulmonary embolism: current status and future potential. J Am Coll Cardiol 1987;10:96B-104B.

40. Goldhaber SZ, Vaughan DE, Markis JE, et al. Acute pulmonary embolism treated with tissue plasminogen activator. Lancet 1986;2:886-9.

41. Daniels LB, Parker JA, Patel SR, et al. Relation of duration of symptoms with response to thrombolytic therapy in pulmonary embolism. Am J Cardiol 1997;80:184-8.

42. Beydilli İ, Yılmaz F, Sönmez BM, et al. Thrombolytic therapy delay is independent predictor of mortality in acute pulmonary embolism at emergency service. Kaohsiung J Med Sci 2016;32:572-8. 